we are dealing with separate systems. These observations indicate that the hydrogen in the outer parts of the Galaxy may be divided into two components: first, the dense hydrogen concentrations that are usually associated with spiral features, fluctuating widely in density and exhibiting small $(5-10 \mathrm{~km} / \mathrm{sec})$ internal velocity dispersion on a small scale, with larger deviations from strictly circular orbits on a large scale; and second, a thin substrate of gas with rather smooth distribution, exhibiting circular

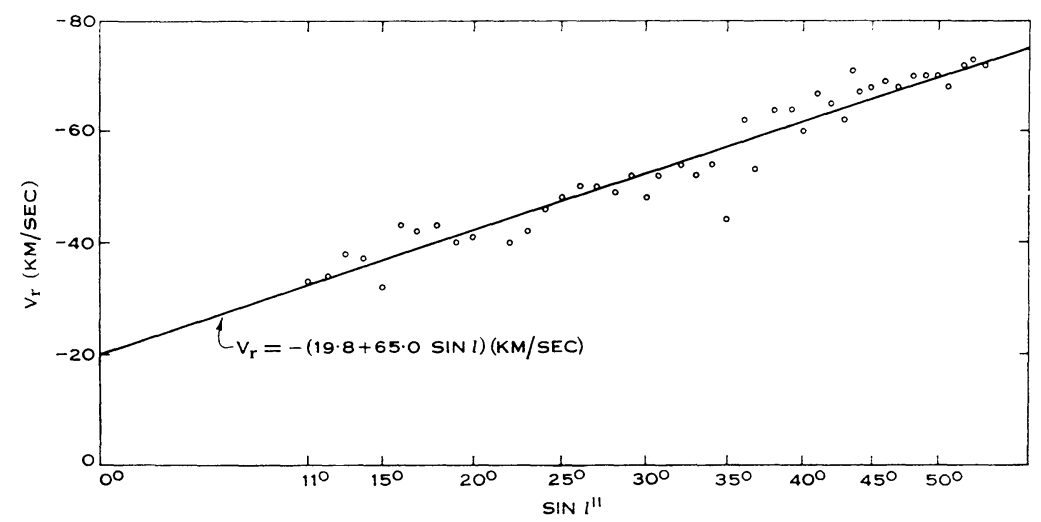

Fig. 4.-Velocity of the $10^{\circ} \mathrm{K}$ contour of antenna temperature with respect to the local standard of rest against $\sin l^{\mathrm{II}}(b=+0.4)$.

motion but with small-scale random motion of at least $20 \mathrm{~km} / \mathrm{sec}$. The substrate is observed over this longitude range on the negative-velocity side, with the random motions of the opposite sign being lost in the more intense radiation of the denser hydrogen concentrations, and hence only a rough estimate of the random internal motions can be given.

\title{
Discussion
}

Oort: In connection with the regular substratum of neutral hydrogen which Dr. Burke indicated, I wish to remark that Mr. Shane in Leiden found an indication of something that might be similar. He observed that in his section perpendicular to the Milky Way at $l^{\text {II }}$ between 22 and $42^{\circ}$ the outer contours were in general much smoother than the higher temperature contours. His outer contours, however, are much fainter than those considered by Dr. Burke, viz. between 2 and $4^{\circ} \mathrm{K}$.

\section{THE MAGNETIC FIELD OF THE GALAXY DETERMINED FROM THE ZEEMAN SPLITTING OF THE 21-CM HYDROGEN LINE}

\section{R. D. Davies}

\section{Nuffield Radio Astronomy Laboratories, Jodrell Bank}

The Zeeman effect can be used to measure directly the longitudinal component of the magnetic field in interstellar neutral hydrogen clouds. The frequency separation between the two circularly polarized components is $28 \mathrm{c} / \mathrm{s}$ for $10^{-5} \mathrm{G}$ and can be inferred from measurements of the intensity difference between left- and right-hand circular polarization as a function of frequency. Earlier experiments at Jodrell Bank 
showed that the mean field in the interstellar medium was less than $10^{-5} \mathrm{G}$ (Davies et al. 1960). Recent work using more sensitive techniques has provided a positive measurement of a weak general magnetic field and of fields of varying intensity in different interstellar clouds.

In experiments in 1960, 1961, and 1962 (Davies et al. 1963) using various radio frequency-switching techniques and a $5 \mathrm{kc} / \mathrm{s}$ bandwidth receiver with a mixer first stage, the Zeeman splitting in the deep absorption features in Cassiopeia A, Cygnus A, Taurus A, and Sagittarius A was investigated. The experimental curves were then used to obtain the longitudinal component of the field passing through an absorption feature (which may consist of contributions from several clouds) by obtaining the bestfit field. This field is calculated by obtaining the best-fitting amplitude of the expected

TABLE 1

THE LONGITUDINAL COMPONENT OF MAGNETIC FIELD FOUND IN VARIOUS ABSORPTION FEATURES

\begin{tabular}{|c|c|c|}
\hline & Feature & $\begin{array}{l}\text { Longitudinal Component } \\
\text { of Magnetic Field } \\
\text { (best fit } \pm 1 \cdot 96 \text { S.D.) } \\
(\mathrm{G})\end{array}$ \\
\hline $\begin{array}{l}\text { Cassiopeia A } \\
\text { Taurus A }\end{array}$ & $\begin{array}{l}-0 \cdot 8 \mathrm{~km} / \mathrm{sec} \text { (Orion arm) } \\
-38 \cdot 1 \mathrm{~km} / \mathrm{sec} \text { (Perseus arm A) } \\
-48 \cdot 2 \mathrm{~km} / \mathrm{sec} \text { (Perseus arm B) } \\
+10 \cdot 3 \mathrm{~km} / \mathrm{sec}\end{array}$ & $\begin{array}{l}-4 \cdot 3 \pm 3 \cdot 8 \times 10^{-6} \\
+4 \cdot 3 \pm 9 \cdot 2 \times 10^{-6} \\
-6 \cdot 7 \pm 3 \cdot 0 \times 10^{-6} \\
+6 \cdot 4 \pm 4 \cdot 5 \times 10^{-6}\end{array}$ \\
\hline
\end{tabular}

Zeeman curve and converting this to a field strength from a knowledge of the shape of the absorption line. On the deep narrow features in Cassiopeia A and Taurus A, which are the most sensitive for measuring the Zeeman effect, the agreement in the various experiments performed under different conditions was good. The results of this series of experiments are given in Table 1 where the best-fitting field and an error equal to the $95 \%$ probability level are tabulated. The best-fitting component is nowhere greater than $7 \times 10^{-6} \mathrm{G}$ and the components for the Perseus B feature of Cassiopeia A and the narrow Taurus $A$ feature appear to be significant.

On the assumption of a circular galactic magnetic field passing along the spiral arms, these longitudinal components can be combined to give an estimate of the strength of such a field. Its value is $-6 \cdot 8 \times 10^{-6} \mathrm{G}$; the negative sign refers to a magnetic field directed in an anti-clockwise direction around the Galaxy when viewed from the north galactic pole. An alternative model which can be fitted to the observational data is a field of $-10 \times 10^{-6} \mathrm{G}$ inclined at $20^{\circ}$ to a tangent to the circular galactic structure. The line of sight is perpendicular to this field configuration at $l^{\mathrm{I}} \sim 120^{\circ}$.

The most recent Zeeman measurements (Davies, Verschuur, and Wild 1962) using a parametric amplifier with a system noise of $200^{\circ} \mathrm{K}$ and six $3 \mathrm{kc} / \mathrm{s}$ bandwidth channels were designed to investigate the magnetic field in individual clouds seen 


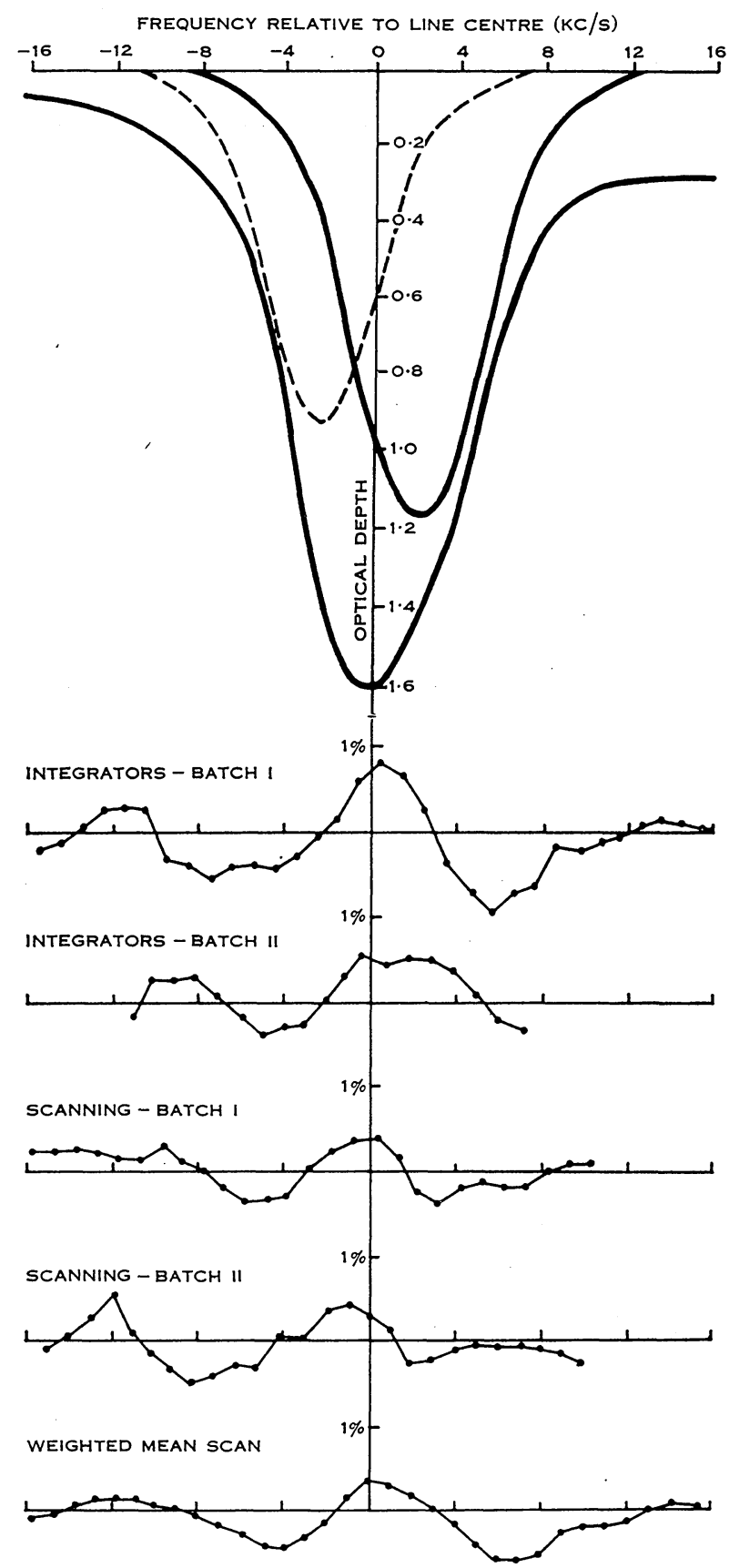

Fig. 1. - Upper part: The absorption profile of the deep feature of Taurus A plotted in terms of optical depth. This profile can be resolved into contributions from two clouds as shown.

Lower part: Four independent batches of Zeeman data which are plotted as the difference between left and right circular polarization intensity expressed as a percentage of the absorption profile depth. The bottom curve is a weighted average of these four curves; the weighting takes into account the expected signal-to-noise ratio for the different batches of data. 
in absorption and emission. The hydrogen-line profiles of these clouds have been analysed in detail by Shuter and Verschuur (private communication). This Zeeman experiment, which also measured the difference in intensity between the right- and left-hand circular components, was performed in two ways. The first used many repetitions of the frequency scanning technique already described while the second employed integration at a fixed frequency. The results of the measurements of the deep feature in Taurus A are shown in Figure 1, where four independent batches of data, two obtained with each method, illustrate the repeatability of the Zeeman effect observed. Similar although somewhat weaker effects were detected in the absorption features of Cassiopeia A and Cygnus A.

Before magnetic fields can be assigned to each cloud the absorption profiles must first be resolved into their various cloud components. In general each cloud will have a different magnetic field passing through it. The expected Zeeman record for a given combination of fields in the components was derived by first calculating the right- and left-hand optical depth curves for the sum of the components and then converting these to brightness temperature curves before performing the subtraction. This analysis has been completed for the Taurus A narrow feature, which was found to be double, and it was found that the Zeeman curve is best fitted by a field of $1.4 \times 10^{-5} \mathrm{G}$ in the high frequency component and $0.8 \times 10^{-5} \mathrm{G}$ in the low frequency component. However, it is permissible in the present experiment to add to or subtract from the observed Zeeman profile a small component of arbitrary amplitude with the same shape as the absorption profile. In consequence the relative strengths of the two components can be altered somewhat but the magnitudes will remain comparable to those given above. The result originally published for this feature (Davies, Verschuur, and Wild 1962) was the best fit for a field only in the high frequency component. Reduction of the rest of the data which extended further from the line centre indicated that the results were better fitted by oppositely directed fields in the two clouds.

A provisional analysis of the results of the Cassiopeia A-Orion arm feature indicates that a longitudinal field of $\leqslant 3 \times 10^{-6} \mathrm{G}$ passes through the two major clouds in this feature. The analysis of the Zeeman measurements on other absorption and emission features in terms of magnetic fields in individual clouds is in progress.

The data at present available already indicate a variability of $5: 1$ in the longitudinal component of the magnetic field in various clouds, which is most likely due to differences in the magnitude of the field in different clouds. Such a variability in the field strength could arise from the compression of the galactic magnetic field in clouds at different stages of compression. With the small but nevertheless effective amount of electrons in the interstellar gas, the magnetic field is bound to the gas and is compressed with it. On this picture the general magnetic field in the Galaxy will be equal to or less than the smallest values measured, namely, $\leqslant 3-5 \times 10^{-6} \mathrm{G}$ when allowance is made for the inclination of the line of sight to the magnetic field.

The present results using the information derived from Zeeman studies of the broad features, as well as of individual clouds, suggest that the general magnetic field of the Galaxy has a value of $5-7 \times 10^{-6} \mathrm{G}$. The relevance of this result to the understanding of various galactic phenomena will now be summarized. With a field 
of this magnitude the polarization of starlight can be adequately explained by the Davis-Greenstein (1951) theory if the uncertainties of the theory are taken into account. On certain theories of galactic structure (Chandrasekhar and Fermi 1953; Hoyle and Ireland 1961) it is possible to hold gas within spiral arms and to preserve the spiral structure of the Galaxy with fields of $\sim 7 \times 10^{-6} \mathrm{G}$. On the other hand, the observed intensity of synchrotron radio emission from the disk of the Galaxy requires a general field of $\sim 3 \times 10^{-5} \mathrm{G}$ (Biermann and Davis 1960; Woltjer 1961) as estimated from the flux of cosmic ray electrons actually measured. An alternative picture of the origin of synchrotron emission is suggested in which the emission comes from localized regions of enhanced magnetic field and perhaps also of high cosmic ray electron flux. These regions are probably old supernova remnants or similar objects. Finally, a field of $\sim 7 \times 10^{-6} \mathrm{G}$ in the disk and let us say $\sim 2 \times 10^{-6} \mathrm{G}$ in the halo is insufficient to hold the most energetic cosmic ray particles with energies $\geqslant 2 \times 10^{18} \mathrm{eV}$ in the Galaxy.

\section{References}

Biermann, L., and Davis, L. (1960).-Zs. f. Ap. 51 : 19-31.

Chandrasekhar, S., and Fermi, E. (1953).-Ap. J. 118: 113-15.

Davies, R. D., ET AL. (1963).-M. $N$. (in press.)

Davies, R. D., Slater, C. H., Shuter, W. L. H., and Wild, P. A. T. (1960).-Nature 187 : 1088-9.

Davies, R. D., VerschuUr, G. L., and Wild, P. A. T. (1962).-Nature 196: 563.

Davis, L., and Greenstein, J. L. (1951).-Ap. J. 114: 206-40.

Hoyle, F., and Ireland, J. G. (1961).-M. N. 122: 35-9.

WOLTJER, L. (1961).-Ap. J. 133 : 352-4.

\section{Discussion}

Little: I should like to ask how you determined the magnitude of the noise level which you indicated on the slide showing the records of the Zeeman effect.

Davies: The noise level was determined from records taken away from the frequency of the absorption and emission lines.

Kerr: These are very difficult observations, and it would be wise to exercise caution until a second group report a positive result. We plan to try the experiment ourselves at a later date. I would be interested to know what results would be obtained under completely different conditions, e.g. an arbitrary region of the sky without a source. Has this comparison been made?

Davies: Measurements were made on a variety of emission and absorption features of different intensity; the noise level measured in the different cases was consistent. The largest Zeeman effects observed were 10 times this r.m.s. noise level.

Roberts: Which results were positive and which were upper limits? Also how do you account for the fact that the published value for Taurus $A$ was greater $\left(2 \cdot 5 \times 10^{-5} \mathrm{G}\right)$ than the largest value quoted?

Davies: Complete analysis of the more extensive scanning records in addition to the published integrator records showed that the Taurus $A$ results are better fitted by a field of $15 \times 10^{-6} \mathrm{G}$ in the high-frequency cloud.

Christiansen: We seem to have reached a limit with the technique used at Jodrell Bank and improved by a factor of 100 over the years; with this perfected technique the results are still doubtful. I think that we need a new technique to produce thoroughly convincing results.

Davies: The largest effects observed are repeatable and are 10 standard deviations. We hope to improve the sensitivity by controlling known systematic effects. 
Parijsky: We may be able to obtain an independent value of magnetic field from the Faraday rotation method provided we know the following quantities:

1. A measure of the emission from HII radio continuum or from radio absorption measurements.

2. The relative intensity for the components of the $3727 \AA$ line for [OII].

The latter seems to be very difficult.

Davies: The former gives a measure of the product $N_{\mathrm{e}}^{2} L$ integrated over the line of sight and it is very difficult to reconstruct the required product $N_{\mathrm{e}} L$ which is required to derive $\mathrm{H}$ from measurements of Faraday rotation.

van Damme: I read in the abstract: "One of the strongest Zeeman effects was measured in the narrow feature of the Taurus A absorption spectrum".

In connection with the remark of Dr. Kerr to attack instrumental effects, may I ask, can you tell how many times you actually have measured this feature to overcome statistical effects?

Davies: Six independent measurements each with effective integration times of more than $1 \mathrm{hr}$ were used; each showed the same polarization effect.

Bolton: It is quite clear from the signal/noise ratio of the diagrams which Davies has shown and the attacks of the radio astronomers that this result is extremely suspect. This is an extremely difficult experiment and it is doubtful, even if the present results are correct, whether the measurements could be extended to give a general picture of the magnetic field of the Galaxy. Much more promising are the Faraday rotation type measurements which, although involving integration along the line of sight, are not subject to experimental doubts and difficulties.

Davies: Such Faraday rotation experiments will give the direction of the field and will give the magnitude only if we know the distribution of electrons in the line of sight; this latter distribution is extremely difficult to determine.

Parijsky: It is very interesting to compare the field determined from 21 -cm measurement with that obtained with the Faraday rotation technique. Let us consider the Crab nebula as an example. It is quite possible that the Faraday rotation occurs in the interstellar medium and not in the nebula itself. The serious support came from the high-resolution observations at Pulkovo in polarized emission at 3 and $6 \cdot 4 \mathrm{~cm}$. Then it is easy to show that the sign of the magnetic field coincides for both methods.

\title{
34. OBSERVATION AND INTERPRETATION OF OPTICAL AND RADIO POLARIZATION
}

\author{
G. WESTERHOUT \\ University of Maryland
}

Measurements of polarization of radio emission from the galactic background were started in 1957 and seemed to be partially successful, but so uncertain that not much weight was attached to them. Only in the last two years have such polarization measurements been fully successful. They were carried out by radio astronomers at Leiden, Cambridge, and Sydney. I shall report here on the measurements made by the Leiden group, and draw some general conclusions. The first year of observations was described by Westerhout et al. (1962) and Brouw, Muller, and Tinbergen (1962); the work has been continued since by Muller, Brouw, Tinbergen, and Berkhuysen (1963). The observations made by the Cambridge group are substantially in agreement with the Leiden work (Wielebinski, Shakeshaft, and PaulinyToth 1962), and so are the Sydney observations, on which Dr. Gardner reports in another paper (this volume, paper 35 ). 